

https://doi.org/10.31533/pubvet.v13n10a440.1-6

\title{
Perfil e epidemiologia da hanseníase em humanos em Alagoas nos anos 2016 e 2017
}

\author{
Camila Maria Freitas da Silva ${ }^{1}$, Carlos Miguel Azarias dos $\operatorname{Santos}^{2}{ }^{\circ}$, Lyara Suzhanny de \\ Oliveira Santos $^{1}{ }^{\bullet}$, Jéssika Silva de $\operatorname{Lima}^{3} \theta$, Josicleide Fernandes de Souza Santos ${ }^{4}{ }^{\bullet}$, Douglas \\ de Oliveira Subrinho ${ }^{4} \theta$, Tiago Ferreira Dantas ${ }^{\circ}{ }^{\circ}$, Carla Fernanda de Oliveira $^{6}{ }^{\theta}$, Taise dos \\ Santos Piancó $^{60}$, Josefa Eleusa da Rocha ${ }^{7} \bullet$ \\ ${ }^{1}$ Graduanda do curso de ciências biológicas, Universidade Estadual de Alagoas - UNEAL, Arapiraca-AL Brasil. \\ ${ }^{2}$ Graduando em ciências biológicas e pesquisador do grupo de extensão, Universidade Estadual de Alagoas - UNEAL e medicina \\ veterinária, Faculdade Regional da Bahia - UNIRB, Arapiraca-AL Brasil.; \\ ${ }^{3}$ Graduada em ciências biológicas, Universidade Estadual de Alagoas - UNEAL e mestranda em Biologia Animal, Universidade Federal \\ dos Vales do Jequitinhonha e Mucuri-UFVJM, Diamantina-MG, Brasil.; \\ ${ }^{4}$ Graduado(a) em Enfermagem, Centro Universitário Cesmac, Arapiraca-AL Brasil. \\ ${ }^{5}$ Graduado em Enfermagem, Universidade Federal de Alagoas - UFAL, Arapiraca-AL Brasil. \\ ${ }^{6}$ Graduada em Zootecnia, Universidade Federal de Alagoas - UFAL, Arapiraca-AL Brasil. \\ ${ }^{7}$ Professora e pesquisadora, do Curso de ciências biológicas, Universidade Estadual de Alagoas - UNEAL, Arapiraca-AL Brasil. \\ *Autor para correspondência: carllos_miguel1@ hotmail.com
}

Resumo. A hanseníase é uma enfermidade infectocontagiosa de evolução crônica que acomete o homem, sendo de difícil diagnóstico e tempo de incubação prolongado, variando de 2 a 10 anos, a metodologia desenvolvida no presente trabalho foi a partir dos dados do Sistema de Informação de Agravos de Notificação (SINAN) para identificar o perfil epidemiológico de pessoas acometidas pela hanseníase no estado de Alagoas nos anos de 2016 - 2017, analisado as variáveis como sexo, faixa etária, modo de detecção, avaliação diagnóstico, avaliação cura, lesões cutâneas, forma clínica, nervos afetados, classificação operacional. Houve uma redução significativa quanto ao percentual de casos de pessoas com hanseníase em $2016 \mathrm{com} 72 \%$ e $2017 \mathrm{com} 28 \%$. As maiores faixas etárias foram 3549, 50-64 e 20-34, representados respectivamente. As diferenciações entre os sexos são relativamente nítidas quanto ao maior número de casos, embora acometa ambos os sexos se observam predominância do sexo masculino com $53 \%$ e o sexo feminino com $47 \%$ dentre outras diversidades de dados referentes à hanseníase. Conclui-se que a utilização dos dados secundários, foi possível realizar a análise epidemiológica e perfil dos infectados com hanseníase no estado de Alagoas, a hanseníase é transmitida principalmente pelas vias aéreas superiores e tem tratamento e cura.

Palavras-chave: doença, epidemiologia, Mycobacterium leprae

\section{Profile and epidemiology of leprosy in human in Alagoas from 2016 to 2017}

Abstract. Leprosy is a chronic contagious infectious disease that affects man, being difficult to diagnose and prolonged incubation time, ranging from 2 to 10 years, the methodology developed in the present study was based on data from the Health Information System Report (SINAN) to identify the epidemiological profile of people affected by leprosy in the state of Alagoas in the years 2016 - 2017, analyzing variables such as gender, age group, mode of detection, diagnostic evaluation, cure evaluation, skin lesions, clinical form, affected nerves, operational classification. There was a significant reduction in the percentage of leprosy cases in 2016 with $72 \%$ and 2017 with $28 \%$. The largest age groups were 35-49, 50-64 and 20-34, respectively. The differences between the sexes are relatively 
clear regarding the largest number of cases, although affecting both sexes, there is a predominance of males with $53 \%$ and females with $47 \%$, among other data diversity related to leprosy. It is concluded that the use of secondary data, it was possible to carry out the epidemiological analysis and profile of leprosy infected in the state of Alagoas, leprosy is transmitted mainly by the upper airways and has treatment and cure.

Keywords: disease, epidemiology, Mycobacterium leprae

\section{Perfil y epidemiología de la lepra en Alagoas en 2016 y 2017}

Resumen. La lepra es una enfermedad infectocontagiosa crónica que afecta al hombre, es difícil de diagnosticar y tiempo de incubación prolongado, que varía de 2 a 10 años, la metodología desarrollada en el presente estudio se basó en datos del Sistema de Informação de Agravos de Notificação (SINAN) para identificar el perfil epidemiológico de las personas afectadas por la lepra en el estado de Alagoas en los años 2016 - 2017, analizando variables como género, grupo de edad, modo de detección, evaluación de diagnóstico, evaluación de curación, lesiones cutáneas, forma clínica, nervios afectados, clasificación operativa. Hubo una reducción significativa en el porcentaje de casos de lepra en 2016 con $72 \%$ y 2017 con 28\%. Los mayores grupos de edad fueron 35-49, 50-64 y 20-34, respectivamente. Las diferencias entre los sexos son relativamente claras con respecto al mayor número de casos, aunque afecta a ambos sexos, hay un predominio de hombres con $53 \%$ y mujeres con $47 \%$, entre otras diversidades de datos relacionados con la lepra. Se concluye que, con el uso de datos secundarios, fue posible realizar el análisis epidemiológico y el perfil de la lepra infectada en el estado de Alagoas, la lepra se transmite principalmente por las vías respiratorias superiores y tiene tratamiento y cura.

Palabras clave: enfermedad, epidemiología, Mycobacterium leprae

\section{Introdução}

A hanseníase é uma doença infectocontagiosa de progresso crônico que prejudica o homem. É uma doença de difícil diagnóstico e tempo de incubação prolongado, variando de 2 a 10 anos; elevação do contágio e diminuição da morbidez. Essa patologia é causada pelo parasita intracelular obrigatório Mycobacterium leprae (bacilo de Hansen). O contágio ocorre via uma pessoa infectada pelo bacilo na forma multibacilar não tratada, sendo expelidas por meio das vias aéreas superiores, transmitindo para indivíduos susceptíveis à doença (Monteiro et al., 2017).

A Organização Mundial de Saúde - OMS define como caso de hanseníase quando o indivíduo mostra um ou mais dos sinais e sintomas: lesão de pele com mudança de sensibilidade; espessamento de nervo juntamente mudança de sensibilidade, existindo ou não história epidemiológica (Lockwood, 2002).

O diagnóstico da hanseníase é definido pela especificação operacional dos acontecimentos. Esta se toma como sustentação a quantidade de lesões na pele, até cinco ou maior que cinco lesões, identificando, na devida ordem, como formas paucibacilar (PB) e multibacilar (MB). Quando executada a baciloscopia sendo positivo o resultado, o caso é notificado como MB livremente da quantidade de lesões. O exame sendo negativo não descarta o diagnóstico de hanseníase nem denomina o caso como PB. A classificação operacional orienta o esquema do tratamento poliquimioterápico a ser determinado para o paciente (Monteiro et al., 2017).

Mycobacterium leprae, agente etiológico da hanseníase. É um parasito intracelular obrigatório, com atração por células cutâneas e células dos nervos periféricos (BRASIL, 2009), se insere no organismo do indivíduo infectado, conseguindo se multiplicar. A multiplicação do bacilo é devagar, conseguindo ficar, por volta, de 11 a 16 dias. $\mathrm{O} M$. leprae tem grande infectividade e pouca patogenicidade, ou seja, infecta diversos indivíduos, portanto poucas pessoas adoecem. Uma vez infectado, o período de incubação é duradouro, com variação de 2 a 7 anos (média de cinco anos) (Barbieri \& Marques, 2009). 
O homem é relatado como única fonte de infecção (reservatório), ainda que também tenham sido encontrados animais espontaneamente infectados. O contágio se dá por meio de um indivíduo infectado, portador do bacilo de Hansen, não examinada, que o expele para o meio exterior, transmitindo para indivíduos susceptíveis. A fundamental via de eliminação do bacilo, pela pessoa doente de hanseníase e a mais plausível porta de entrada no organismo passível de ser infectados são as vias aéreas superiores, o trato respiratório (BRASIL, 2009).

O princípio essencial do controle da hanseníase é controlar a morbidade, ou seja, é a forma da detectar novos casos, tratar com o esquema de poliquimioterápico (PQT), prevenir de incapacidades e reabilitação. Por tanto, se faz necessário garantir que as ações de controle da doença sejam ligadas as incumbências da Atenção Primária à Saúde (Lanza \& Lana, 2011). Desta forma, diante do abordado, este estudo visou apresentar características epidemiológicas, diagnósticos clínicos e distribuição espacial de casos de hanseníase no estado de Alagoas entre os anos de 2016 e 2017. Tendo por objetivo analisar o perfil das pessoas com Hanseníase, facilitando a identificação do público alvo. Portanto a partir das subnotificações houve interesse no estudo, por se tratar de uma doença reemergente por sua vez ainda muito negligenciada nas formas de detecção e ao realizar as notificações.

\section{Metodologia}

Trata-se de um estudo descritivo, epidemiológico, retrospectivo com abordagem quantitativa analítica, realizado com dados do Sistema de Informação de Agravos de Notificação (SINAN, 2018) para identificar o perfil epidemiológico de pessoas acometidas pela hanseníase no estado de Alagoas. A pesquisa epidemiológica documental estudada em plataforma digital por meio do Google Acadêmico e no site SINAN (2018).

Para este artigo utilizou-se como critério de inclusão pacientes diagnosticados com hanseníase, notificados e residente no estado de Alagoas referido aos anos de 2016 - 2017. Analisando as variáveis como sexo, faixa etária, modo de detecção, avaliação diagnóstico, avaliação cura, lesões cutâneas, forma clínica, nervos afetados, classificação operacional.

Os dados referentes ao estado de Alagoas foram disponibilizados pela secretaria municipal de saúde de Arapiraca, departamento de vigilância epidemiológica, a montagem de gráficos e tabelas quanto as organizações dos mesmos foram realizadas por meio do pacote office, Microsoft Excel.

\section{Resultados e discussão}

No período de 2016 a 2017, foram notificados 442 casos de hanseníase no estado de Alagoas, com média de 221 casos anualmente. A maior frequência observada foi no ano de $2016(\mathrm{n}=319$ casos, $72 \%)$ e a menor ocorrência foi registrada no ano de $2017(\mathrm{n}=123$ casos, 28\%) (Figura 1). Percebe-se que houve uma diminuição entre um e outro ano, resultado semelhante obtido por Barbosa et al. (2014).

Com relação à ocorrência de casos por sexo, a Figura 2 mostra que 236 casos (53\%) eram de indivíduos do sexo masculino, sendo este grupo mais prevalente quando comparado ao do sexo feminino. Tal resultado é condizente com a literatura, em que esta apresenta vários estudos divergentes em relação à prevalência de hanseníase quanto ao sexo (Lima et al., 2010). Alguns autores comprovam que o maior contato social entre homens e sua frequente exposição a ambientes de risco favorece o aumento do número de casos, enquanto que a menor preocupação com a estética corporal e a falta de políticas específicas para esse grupo pode contribuir na deficiência do diagnóstico, o que justificaria o predomínio do sexo feminino em alguns estudos (Melão et al., 2011; Silva et al., 2010).

Sobre a distribuição da hanseníase por faixa etária, avaliaram-se as seguintes classificações: 01-04 anos, 05-09, 10-14, 15-19, 20-34, 35-49, 50-64, 65-79 e 80 ou mais anos (Tabela 1). A faixa etária predominante, registrando-se maior número de casos entre 35 e 49 anos, com 121 casos. De acordo com os dados levantados quanto à faixa etária, o estudo revela que a hanseníase atinge predominantemente os adultos. Resultado semelhante foi observado por Barbosa et al. (2014), no qual os autores em seu 
estudo apresentaram concentração de idades entre 20 e 39 anos. Esse intervalo relaciona-se com o período economicamente produtivo e caracteriza a hanseníase como doença de adultos e idosos.

A distribuição dos casos, segundo a classificação operacional, foi classificada como maior quantidade no total de multibacilares para os anos de 2016 e 2017 somados ( $\mathrm{n}=276$ casos) e paucibacilares para ( $\mathrm{n}=166$ casos). Quanto ao número de lesões cutâneas, a maioria dos casos $(\mathrm{n}=202)$ apresentava de 2 a 5 lesões. Observou-se que a maioria dos indivíduos apresentou de duas a cinco lesões cutâneas. Resultados semelhantes foram apresentados no estudo de Ribeiro Júnior et al. (2012), no qual todos os pacientes estudados apresentaram o mesmo número de lesões cutâneas, reportados por este estudo.



2016

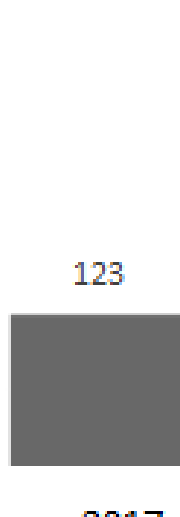

2017

Figura 1. Distribuição do número de casos notificados de hanseníase no estado de Alagoas entre os anos de 2016 a 2017. Fonte: Sistema de Informação de Agravos de Notificação - SINAN NET (2018).

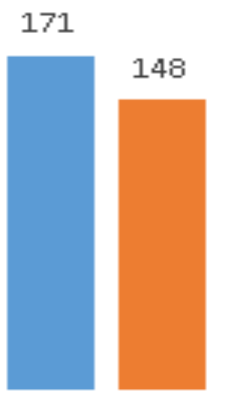

2016

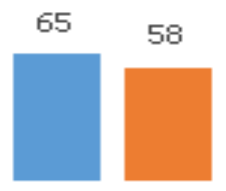

2017
Figura 2. Distribuição por sexo de casos de hanseníase confirmados em residentes do estado de alagoas entre os anos de 2016 a 2017. Fonte dos dados: Sistema de Informação de Agravos de Notificação SINAN NET (2018).

A hanseníase, além de ser um problema de saúde pública devido ao grande número de casos, tem um alto potencial de causar incapacidades físicas, interferindo na fase produtiva e na vida social do paciente, determinando perdas econômicas e traumas psicológicos. Essas incapacidades têm sido ponderadas pelo estigma e pela discriminação dos pacientes, com tudo cabe a importância de sempre procurar um médico, a forma de detecção em maior quantidade deu-se ao modo de encaminhamento tendo soma dos dois anos um total de 203 pacientes, quanto a demanda espontânea dá-se 123 pacientes, sendo valores significativos. Pereira et al. (2011) relatam que uma das formas mais eficazes de determinar se o diagnóstico tem sido precoce é identificando a presença de incapacidades físicas no momento da diagnose.

Tabela 1. Distribuição por faixa etária de pacientes com hanseníase segundo o sexo, no estado de Alagoas entre os anos de 2016 a 2017.

\begin{tabular}{lccrr}
\hline Faixa Etária SINAN & 2016 & 2017 & $2016 \%$ & $2017 \%$ \\
\hline $01-04$ & 0 & 1 & $0 \%$ & $100 \%$ \\
$05-09$ & 4 & 2 & $67 \%$ & $33 \%$ \\
$10-14$ & 13 & 9 & $59 \%$ & $41 \%$ \\
$15-19$ & 11 & 3 & $79 \%$ & $21 \%$ \\
$20-34$ & 80 & 25 & $76 \%$ & $24 \%$ \\
$35-49$ & 87 & 34 & $72 \%$ & $28 \%$ \\
$50-64$ & 82 & 34 & $71 \%$ & $29 \%$ \\
$65-79$ & 36 & 15 & $71 \%$ & $29 \%$ \\
$80-+$ & 6 & 0 & $100 \%$ & $0 \%$ \\
\hline Total & 319 & 123 & $72 \%$ & $28 \%$ \\
\hline For & & & $71 \%$ \\
\hline
\end{tabular}

Fonte: Sistema de Informação de Agravos de Notificação - SINAN NET (2018). 
Tabela 2. Características clínicas e epidemiológicas de pacientes com hanseníase no estado de Alagoas entre os anos de 2016 a 2017. (n) = Número de casos e $*=$ sem dados.

\begin{tabular}{|c|c|c|}
\hline Características & $2016, \mathrm{n}$ & $2017, \mathrm{n}$ \\
\hline \multicolumn{3}{|c|}{ Classificação Operacional } \\
\hline Multibacilar & 201 & 75 \\
\hline Paucibacilar & 118 & 48 \\
\hline \multicolumn{3}{|l|}{ Número de Lesões } \\
\hline Lesão Única & $*$ & $*$ \\
\hline 2 a 5 Lesões & 155 & 47 \\
\hline$>5$ Lesões & 48 & 14 \\
\hline Sem informação & 116 & 62 \\
\hline \multicolumn{3}{|l|}{ Modo de Detecção } \\
\hline Encaminhamento & 149 & 54 \\
\hline Demanda Espontânea & 94 & 29 \\
\hline Exame Coletividade & 8 & 6 \\
\hline Exame Contato & 10 & 6 \\
\hline Outros Modos & 6 & 1 \\
\hline Sem informação & 52 & 27 \\
\hline \multicolumn{3}{|l|}{ Avaliação de Cura } \\
\hline$\overline{\text { Grau } 0}$ & 51 & 3 \\
\hline Grau 1 & 16 & 0 \\
\hline Grau 2 & 3 & 2 \\
\hline Não Avaliado & 6 & 1 \\
\hline Branco & 243 & 117 \\
\hline \multicolumn{3}{|l|}{ Avaliação e Diagnostico } \\
\hline Grau 0 & 152 & 56 \\
\hline Grau 1 & 86 & 26 \\
\hline Grau 2 & 30 & 8 \\
\hline Não Avaliado & 27 & 17 \\
\hline Branco & 24 & 16 \\
\hline \multicolumn{3}{|l|}{$\overline{\text { Nervos Afetados }}$} \\
\hline Menor e igual 5 & 70 & 15 \\
\hline Maior que 5 & 2 & 2 \\
\hline Ignorado & 247 & 106 \\
\hline \multicolumn{3}{|l|}{ Formas Clínicas } \\
\hline Indeterminada & 40 & 17 \\
\hline Tuberculóide & 69 & 31 \\
\hline Dimorfa & 82 & 32 \\
\hline Virchowiana & 62 & 15 \\
\hline Não Classificada & 36 & 16 \\
\hline Ignorado & 30 & 12 \\
\hline
\end{tabular}

Fonte: Sistema de Informação de Agravos de Notificação - SINAN NET (2018).

\section{Conclusão}

Com a utilização dos dados secundários foi possível realizar a análise epidemiológica e perfil dos infectados com hanseníase no estado de Alagoas. A hanseníase é transmitida principalmente pelas vias aéreas superior, por meio de contato próximo e prolongado de uma pessoa suscetível (com maior probabilidade de adoecer) com uma pessoa doente sem tratamento. Comprovou-se que indivíduos do sexo masculino são os mais acometidos, a fase adulta lidera e na sequência os idosos apresentam a patologia também. A partir dos resultados propostos pela pesquisa, pode-se observar que ao decorrer dos anos, houve uma redução expressiva no número de casos da hanseníase na população estudada, o que demonstra que os programas de controle da doença estão se tornando eficazes possibilitando assim 
inferir que o diagnóstico tem se tornado precoce e as estratégias de prevenção e controle da doença mais eficaz pela notória redução nos números de casos.

\section{Referências bibliográficas}

Barbieri, C. L. A. \& Marques, H. H. S. (2009). Hanseníase em crianças e adolescentes: revisão bibliográfica e situação atual no Brasil. Pediatria, 31(4):281-290.

Barbosa, D. R. M., Araújo, A. A., Damaceno, J. C. F., Almeida, M. G. \& Santos, A. G. (2014). Perfil epidemiológico da hanseníase em cidade hiperendêmica do Maranhão, 2005-2012. Revista Rede de Cuidados em Saúde, 8(1):1-12.

BRASIL. Ministério da saúde. Guia de vigilância epidemiológica. $7^{\text {ed }}$. Brasília: Ministério da Saúde, 2009.

Lanza, F. M. \& Lana, F. C. F. (2011). Descentralização das ações de controle da hanseníase na microrregião de Almenara, Minas Gerais. Revista Latino Americana de Enfermagem, 19(1):187-194.

Lima, H. M. N., Sauaia, N., Costa, V. R. L., Coelho Neto, G. T. \& Figueiredo, P. M. S. (2010). Perfil epidemiológico dos pacientes com hanseníase atendidos em Centro de Saúde em São Luís, MA. Revista Brasileira de Clínica Médica, 8(4):323-327.

Lockwood, D. N. J. (2002). Leprosy elimination - a virtual phenomenon or a reality? Bmj, 324(7352):1516-1518.

Melão, S., Blanco, L. F. O., Mounzer, N., Veronezi, C. C. D. \& Simões, P. W. T. A. (2011). Perfil epidemiológico dos pacientes com hanseníase no extremo sul de Santa Catarina, no período de 2001 a 2007. Revista da Sociedade Brasileira de Medicina Tropical, 44(1):79-84.

Monteiro, M. J. d. S. D., Santos, G. M., Barreto, M. T. S., Silva, R. V. S., Jesus, R. L. R. \& Silva, H. J. N. (2017). Perfil epidemiológico de casos de hanseníase em um estado do nordeste brasileiro. Revista de Atenção à Saúde, 15(54):21-28.

Pereira, E. V. E., Nogueira, L. T., Machado, H. A. S., Lima, L. A. N. \& Ramos, C. H. M. (2011). Perfil epidemiológico da hanseníase no município de Teresina, no período de 2001-2008. Anais Brasilero de Dermatologia, 86(2):235-240.

Ribeiro Júnior, A., F,, Vieira, M. A. \& Caldeira, A. P. (2012). Perfil epidemiológico da hanseníase em uma cidade endêmica no Norte de Minas Gerais. Revista Brasileira de Clínica Médica, 4272-277.

Silva, A. R., Matos, W. B., Silva, C. C. B. \& Gonçalves, E. d. G. R. (2010). Hanseníase no município de Buriticupu, Estado do Maranhão: busca ativa de casos na população adulta. Revista da Sociedade Brasileira de Medicina Tropical, 43(6):691-694.

SINAN. Disponível em: < http://tabnet.datasus.gov.br/cgi/deftohtm.exe?sinannet/cnv/esquistoAL.def> acesso em: 20 de junho de 2018 às 20:00h.

Recebido: 8 de agosto, 2019.

Aprovado: 9 de setembro, 2019.

Publicado: 11 de novembro, 2019.

Licenciamento: Este artigo é publicado na modalidade acesso aberto sob a licença Creative Commons Atribuição 4.0 (CC-BY 4.0), a qual permite uso irrestrito, distribuição, reprodução em qualquer meio, desde que o autor e a fonte sejam devidamente creditados. 